\title{
Ablación con radiofrecuencia del esófago de Barret con displasia 0 carcinoma in situ. Experiencia preliminar
}

\section{Preliminary Experience with Radiofrequency Ablation of Barrett's Esophagus with in situ Dysplasia or Carcinoma}

\author{
Rodrigo Castaño, MD, ${ }^{1}$ Óscar Álvarez, MD, ${ }^{2}$ Amy Piñeres, MD, ${ }^{3}$ Mario H Ruiz, MD, ${ }^{4}$ Andrés Rojas, MD, ${ }^{5}$ Alejandra Álvarez, ${ }^{6}$ \\ Luis Miguel Ruiz, ${ }^{6}$ David Restrepo, ${ }^{7}$ Víctor Calvo. ${ }^{8}$
}

1 Cirugía Gastrointestinal y Endoscopia. Jefe de Postgrado Cirugía General U.P.B, Grupo Gastrohepatología Universidad de Antioquia, Instituto de Cancerología Clínica las Américas. MedellínColombia

2 Gastroenterólogo, Texas Valley Coastal Bend (Veterans Administration) y Clinical Assistant Professor UTHSCSA. Estados Unidos

${ }_{3}^{3}$ Cirujano General, Clínica Bolivariana. MedellínColombia

${ }^{4}$ Cirujano General, Hospital Pablo Tobón Uribe. Medellín-Colombia

5 Cirujano General, Instituto de Cancerología-Clínica las Américas. Medellín-Colombia

${ }^{6}$ Estudiantes de Pregrado, Facultad de Medicina U.P.B. Medellín-Colombia

7 Estudiante de Pregrado, Facultad de Medicina CES. Medellín-Colombia

${ }^{8}$ Estadístico. Medellín-Colombia

Fecha recibido: $\quad 31-03-14$ Fecha aceptado: 05-11-14

\begin{abstract}
Resumen
Introducción: el esófago de Barrett (EB) es el resultado de la injuria crónica del ácido sobre el epitelio esofágico por el RGE. Se define cuando el epitelio metaplásico columnar reemplaza al epitelio escamoso estratificado que normalmente recubre el esófago. El EB representa un factor de riesgo para el adenocarcinoma de esófago. El objetivo de la ablación por radiofrecuencia (RFA) es destruir el epitelio metaplásico con la corriente eléctrica de radiofrecuencia para estimular la reaparición del epitelio plano estratificado del esófago distal.

Objetivo: evaluar la eficiencia y seguridad de la técnica de RFA, recientemente introducida en la ciudad de Medellín, Colombia, para el manejo del EB con displasia o carcinoma in situ.

Materiales y métodos: diez pacientes fueron tratados con RFA. Pacientes con EB con displasia o carcinoma in situ como hallazgo histológico fueron elegidos para el tratamiento. La técnica de RFA se aplicó usando el equipo BARRX: el sistema circular $\mathrm{HALO}^{360}$ o el HALO ${ }^{90}$. Los efectos macro o microscópicos de la terapia con RFA, la tolerancia del paciente al tratamiento así como las complicaciones fueron evaluadas.

Resultados: un grupo de 10 pacientes recibió la terapia con RFA, todos terminaron la terapia y el seguimiento. En siete pacientes había displasia de bajo grado, en dos de alto grado y un paciente con carcinoma in situ. Dos pacientes tenían resección endoscópica con bandas de nódulos de la mucosa previa a la terapia con RFA. Se realizaron un total de 13 procedimientos: 10 con $\mathrm{HALO}^{360}, 2 \mathrm{HALO}^{90}$ y un paciente tratado con argón plasma para un EB residual. En todos los pacientes se logró la erradicación del epitelio esofágico metaplásico y displásico como se confirmó en la evaluación endoscópica e histológica. No se encontraron complicaciones significativas relacionadas con la terapia de RFA, pero en dos pacientes se encontró estenosis del esófago y uno requirió dilataciones endoscópicas tres semanas después de la terapia.

Conclusiones: con base en estos resultados preliminares consideramos que este es un método promisorio, sin mayores complicaciones y bien tolerado por el paciente. En la mayoría de pacientes se erradicó exitosamente el epitelio metaplásico y displásico del esófago distal.
\end{abstract}

\section{Palabras clave}

Esófago de Barrett, cáncer de esófago, displasia en esófago, metaplasia, carcinoma in situ, ablación por radiofrecuencia.

\section{INTRODUCCIÓN}

El esófago de Barrett (EB) es el resultado de una injuria crónica de la mucosa esofágica por el ácido gástrico en el curso de la enfermedad por reflujo gastroesofágico (RGE). El EB se define como la metaplasia intestinal columnar que reemplaza el epitelio escamoso estratificado que normalmente se asienta en el esófago. El EB es una condición de riesgo para la aparición de adenocarcinoma de esófago (ACE). La estadística mundial que evalúa el EB en la población general es divergente y varía de 0,9 a 6\% (1). La tasa anual de incidencia de ACE en pacientes con EB se estima 


\begin{abstract}
Introduction: Barrett's esophagus (BE) is the result of chronic damage to the esophageal epithelium caused by the acid of gastrointestinal reflux diseases (GERD). It is defined as replacement of the by stratified squamous epithelium that normally lines the esophagus with metaplastic columnar epithelium. BE represents a risk factor for esophageal adenocarcinoma. The aim of radiofrequency ablation (RFA) is to destroy the metaplastic epithelium with the electric current of a radiofrequency to stimulate the reappearance of stratified squamous epithelium in the distal esophagus.

Objective: The objective of this study was to evaluate the efficiency and safety of RFA which has recently been introduced in the city of Medellin, Colombia for management of BE with in situ dysplasia or carcinoma.

Materials and Methods: Ten patients were treated with RFA. BE patients with in situ dysplasia or carcinoma and histological diagnoses were chosen for treatment. RFA procedures were done with BARRX equipment, the circular HALO360 system or the HALO90 system. Macroscopic and microscopic effects of RFA, patient tolerance to treatment and complications were evaluated.

Results: A group of 10 patients received treatment with RFA. All ten finished treatment and follow-up. Seven patients had low-grade dysplasia, two had high-grade dysplasia, and one patient had in situ carcinoma. Two patients had undergone endoscopic resections of nodules and treatments of mucosa with bands prior to therapy with RFA. A total of 13 procedures were performed: 10 RFAs with the HALO360, 2 RFAs with the HALO90 and one argon plasma treatment for residual BE in one patient. Eradication of metaplastic and dysplastic esophageal epithelium was achieved in all patients and confirmed by endoscopic and histologic evaluation. No significant complications related to RFA were found, but in two patients esophageal stenoses were found. One required endoscopic dilatation three weeks after therapy.

Conclusions: Based on these preliminary results we believe that this is a promising method that is welltolerated by patients and which has no major complications. Metaplastic and dysplastic epithelium of the distal esophagus were successfully eradicated in most patients.
\end{abstract}

\title{
Keywords
}

Barrett's esophagus, esophageal cancer, esophageal dysplasia, metaplasia, in situ carcinoma, radiofrequency ablation.

en menos de $0,5 \%(2)$; sin embargo, los pacientes de EB con displasia de alto grado progresan a ACE en $6 \%$ por año (3). El incremento en la incidencia del ACE es la mayor que cualquier otra neoplasia en reportes foráneos (4). En vista del pobre reconocimiento endoscópico del EB y del estable crecimiento de los casos de ACE, estos pacientes se han convertido recientemente en un foco de atención en todas las latitudes.

Durante los dos últimos decenios se han implementado diferentes métodos endoscópicos para la erradicación del EB. Los más frecuentes son la terapia con argón plasma (5), la resección endoscópica de la mucosa (6), la disección submucosa (7), la crioterapia (8), la terapia fotodinámica (9) y más recientemente se ha introducido la ablación por radiofrecuencia (RFA) (10) incluso con publicaciones en nuestro medio como la de los doctores Rey \& Pinilla (11).

En la RFA se aplica corriente eléctrica alterna que induce un campo electromagnético cargado de iones que rápidamente oscilan, colisionan uno con otro y crean una fricción molecular que resulta en la liberación de energía exotérmica, que aplicada al tejido esofágico lleva a una lesión térmica controlada con vaporización de agua, coagulación de proteínas y necrosis celular, para destruir el epitelio metaplásico o displásico y así estimular el proceso de rege- neración del epitelio (12). No deben existir alteraciones de contacto entre el esófago y la interfaz de la ablación para que se produzca la erradicación eficaz de la mucosa. Por lo tanto, la RFA generalmente no se aplica al tejido nodular con intención curativa.

En el primer estudio para la erradicación del EB con displasia, ensayo aleatorizado, controlado simulado, multicéntrico, de RFA versus un grupo de control, Shaheen mostró un $77 \%$ versus $2,3 \%$ de erradicación del EB, $81 \%$ versus $19 \%$ en la erradicación de la displasia de alto grado, $90 \%$ versus $23 \%$ en la erradicación de cualquier displasia, $2,4 \%$ versus $19 \%$ en la tasa de progresión al cáncer con un 6\% de estenosis en un seguimiento de 12 meses (13). Recientemente estos investigadores actualizan sus cifras con un seguimiento a largo plazo y demuestran tasas de erradicación de la displasia y la metaplasia intestinal de 98 y 91\% respectivamente, para segmentos largos de EB (media de $5 \mathrm{~cm}$ ) después de 3 años de seguimiento (14).

La evidencia de diferentes estudios bien diseñados, como el citado previamente, aleatorizados, controlados y ciegos (15), sugieren que la RFA es altamente efectiva para remover el EB a la endoscopia e histología con un margen de seguridad favorable (16). Aunque el seguimiento a largo plazo es limitado, en el seguimiento a 5 años la erradicación 
del EB se mantiene en más de $90 \%$ de los pacientes (17). Además, estudios de las propiedades del epitelio neoescamoso que se regenera después de la RFA muestra la ausencia de las anomalías oncogénicas preexistentes, sugiriendo una transición a un epitelio de bajo riesgo (18).

El propósito del presente estudio es evaluar la eficacia y seguridad de la ablación con radiofrecuencia para el manejo del EB con algún grado de displasia y el carcinoma de esófago descrito como in situ en un grupo de pacientes tratados en la ciudad de Medellín, Colombia.

\section{MATERIALES Y MÉTODOS}

La terapia con RFA se realizó a 10 pacientes ( 9 hombres) con edades entre 52 y 74 años (media 66 años) con catéter $\mathrm{HALO}^{360}$, en un periodo de 18 meses (enero de 2012 a julio de 2013). Todos los pacientes tenían antecedentes de reflujo gastroesofágico sintomático por más de 10 años y en todos había hernia hiatal. El criterio para participar en el estudio es la presencia de EB con displasia de cualquier grado o de carcinoma in situ confirmados histológicamente por dos patólogos. En dos pacientes había intervención previa con mucosectomía endoscópica con bandas por nódulos esofágicos con displasia de alto grado y carcinoma in situ. En ninguno de los pacientes se realizó evaluación por ultrasonido endoscópico.

Antes del inicio de la terapia todos los pacientes fueron evaluados con endoscopia con biopsias de los cuatro cuadrantes del esófago, cada cm en la longitud del EB. El EB fue clasificado de acuerdo con la clasificación de Praga (19). El procedimiento se presupuestó para realizar cada dos meses en caso de ser necesario y bajo anestesia general, usando el equipo HALO producido por BARRX Medical (Sunnyvale, California USA). En la totalidad de los pacientes se utilizó la terapia inicial la sonda $\mathrm{HALO}^{360}$ por la presencia de EB circunferencial. Como terapia complementaria se utilizó el dispositivo $\mathrm{HALO}^{90}$ (figura 1).
Antes de la terapia se pasó sobre guía el catéter medidor de $4 \mathrm{cms}$ de longitud, para determinar el diámetro del esófago y facilitar la elección del tamaño del catéter HALO ${ }^{360}$ que se utilizará en la terapia. El catéter de ablación HALO tiene un balón de $3 \mathrm{~cm}$ en su extremo distal con un electrodo bipolar en su superficie externa. Este electrodo está disponible en diámetros del balón de 22, 25, 28, 31, y 34 $\mathrm{mm}$. Estudios de dosimetría han mostrado que la ablación circunferencial es más efectiva con la aplicación de 10 a 12 $\mathrm{j} / \mathrm{cm}^{2}$ y $40 \mathrm{~W} / \mathrm{cm}^{2}$ para lograr la erradicación de todo el espesor de la mucosa $(20,21)$ (figura 2).

La radiofrecuencia se administró al tejido displásico o con carcinoma in situ por un electrodo bipolar a $12 \mathrm{~J} / \mathrm{cm}^{2}$. La profundidad de la ablación debe incluir la mucosa y la porción superficial de la submucosa. En dos de los pacientes se realizó terapia $\mathrm{HALO}^{90}$. En un paciente a quien no se autorizó la terapia complementaria con catéter $\mathrm{HALO}^{90}$ se terminó el tratamiento con argón plasma.

Para cualquiera de los catéteres de ablación $\left(\mathrm{HALO}^{360 / 90}\right)$ el generador de energía automáticamente aplica la radiofrecuencia al electrodo tras la activación con el pedal. Debido a la combinación de alta potencia y energía preestablecidos por el equipo, el resultado es una ablación uniforme en profundidad de penetración del tejido (1000 um) lo que no es operador dependiente. Por lo tanto, la RFA resulta en una destrucción controlada de la capa epitelial columnar, la lámina propia y parte de la capa muscular de la mucosa, mientras que la submucosa permanece ilesa (22).

Después de la terapia el paciente sale con la formulación por un mes de bloqueador de bomba de protones a dosis estándar dos veces al día más un bloqueador ácido oral antes de cada comida y a la hora de acostarse. Además, el paciente sigue con una dieta licuada por 3 días y luego una dieta blanda por una semana.

Se evaluaron los efectos endoscópicos y microscópicos de la terapia así como la tolerancia de los pacientes al procedimiento y las posibles complicaciones.

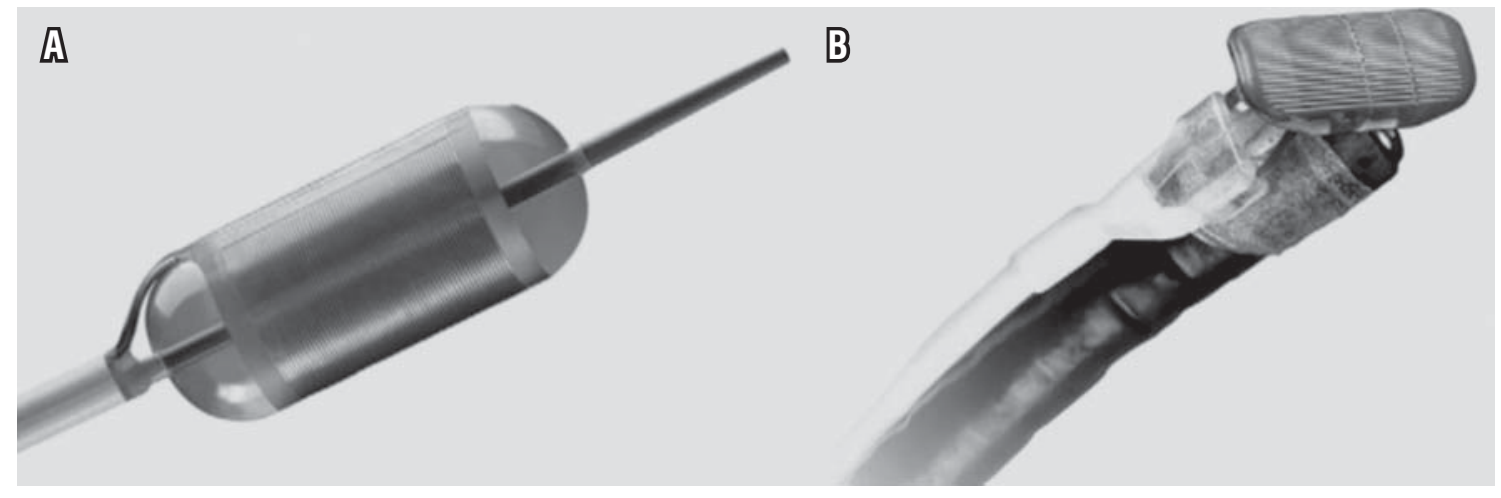

Figura 1. A. Balón de ablación circunferencial para RFA HALO ${ }^{360}$ (BARRX Medical, Sunnyvale, California). B- Equipo de ablación focal para RFA HALO ${ }^{90}$ (BARRX Medical, Sunnyvale, California). 

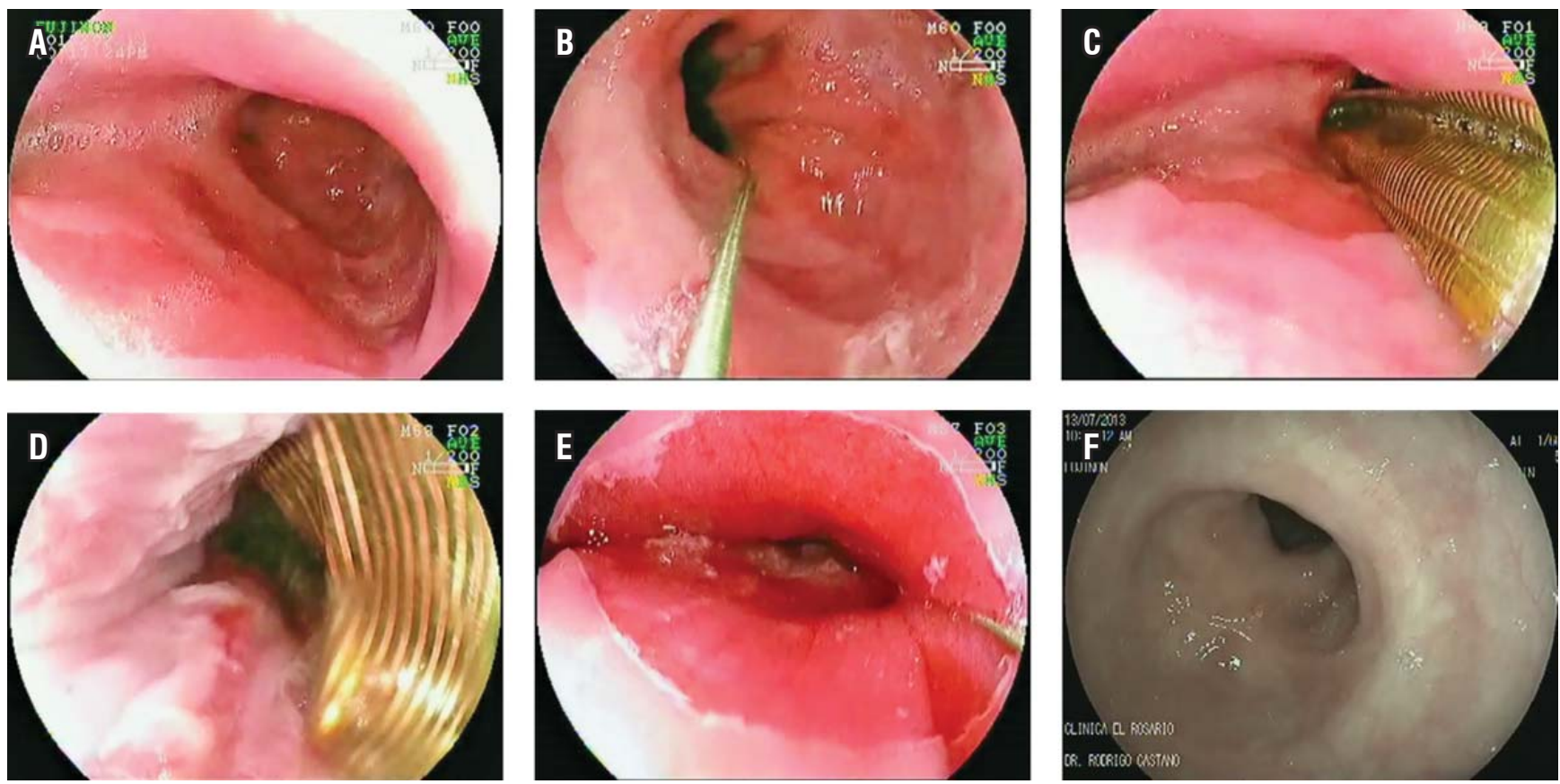

Figura 2. Secuencia fotográfica de la RFA en el esófago de Barrett displásico. A. Esófago de Barrett largo con mucosectomía con bandas previa. B. Paso de la guía para el catéter medidor y de radiofrecuencia. C. Balón de radiofrecuencia ubicado un cm por encima del EB. D. Efectos sobre el EB del catéter de radiofrecuencia $\mathrm{HALO}^{360}$. E. Aspecto del esófago después de la RFA y desbridamiento. F. Tres meses después control con cicatrices tras la terapia con RFA.

Se programa un control endoscópico a los tres, seis y doce meses con biopsias de cuatro cuadrantes del área tratada por RFA. Se hizo especial énfasis en el seguimiento endoscópico e histológico a la reaparición del EB epitelial evidente o al llamado EB sumergido ("Buried Barrett") con la ayuda de la cromoendoscopia digital (FICE o NBI).

\section{RESULTADOS}

De los diez pacientes, en siete había displasia de bajo grado, en dos de alto grado y uno tenía un antecedente de carcinoma in situ. La totalidad de los pacientes fueron tratados en su terapia inicial con la sonda $\mathrm{HALO}^{360}$. El epitelio metaplásico anormal con los cambios displásico y el carcinoma in situ fue erradicado en la totalidad de los pacientes. Dos pacientes requirieron de una sesión más con el catéter $\mathrm{HALO}^{90}$, mientras que en otro paciente con un islote residual, por problemas administrativos, se le trató con argón plasma.

En tres pacientes había intervenciones previas a la RFA, uno de ellos con dos terapias como eran la ablación con argón plasma y una mucosectomía con bandas por DAG, en los otros dos pacientes había una mucosectomía con bandas por carcinoma in situ y una terapia con argón plasma que no interfirieron para el éxito de la terapia con RFA. Solo en una paciente no había hernia hiatal, en los demás la hernia tampoco fue impedimento para una terapia segura.
No se presentaron complicaciones inmediatas atribuibles a la terapia. En las dos semanas siguientes a la intervención los pacientes refirieron dolor torácico espontáneo que se exacerbaba con la ingesta, que mejoró con la medicación ordenada desde el principio; solo en un paciente se presentó fiebre y vómito la noche después de la terapia. Solo en tres pacientes el dolor ameritó una intervención diferente a la instaurada desde el momento en que se realizó la terapia (analgésicos parenterales y/o manejo en urgencias).

En dos pacientes se presentaron estenosis sintomáticas, uno de ellos ameritó a las tres semanas de la terapia dilataciones con bujía de Savary-Guilliard hasta $17 \mathrm{~mm}$, logrando el alivio de la estenosis con solo una sesión de dilatación.

El seguimiento promedio es corto, menor a un año, pero en ninguno de los pacientes se demostró la aparición de epitelio metaplásico o displásico ni carcinoma. En la tabla 1 se recogen las características de los pacientes.

\section{DISCUSIÓN}

A la fecha no existe una indicación consistente y unívoca en el manejo del EB con displasia o del carcinoma in situ del esófago (23). No obstante el creciente interés en el manejo de esta condición, la mayoría de las guías de manejo solo recomiendan en los pacientes con EB el seguimiento para determinar la aparición en forma precoz del cáncer. La 
Tabla 1. Características del grupo de pacientes.

\begin{tabular}{cccccccc}
\hline Sexo & Edad & Histología & BC & BM & Terapias previas & Terapia & Complicación \\
\hline M & 61 & DBG & 3 & 4 & - & HALO360 & - \\
M & 69 & DBG & 2 & 4 & - & HALO360190 & Dolor \\
F & 70 & DAG & 2 & 3 & - & HALO 30190 & Dolor \\
M & 77 & DBG & 2 & 3 & Bandas & HALO360 & - \\
M & 53 & DBG & 1 & 2 & - & HALO360 & - \\
M & 62 & Ca in situ & 3 & 5 & APC, bandas & HALO360, APC & - \\
F & 51 & DAG & 2 & 2 & - & HALO360 & Estenosis-dilatación \\
M & 55 & DBG & 2 & 3 & - & HALO360 & Dolor-fiebre \\
M & 59 & DBG & 2 & 4 & APC & HALO360 & Estenosis \\
M & 74 & DBG & 3 & 4 & - & HALO360 & - \\
\hline
\end{tabular}

frecuencia del seguimiento endoscópico dependerá del tipo de lesiones asociadas al EB según lo informado por los hallazgos histológicos (24). Para la metaplasia aislada la endoscopia con biopsias es recomendable cada 2 a 3 años, para la displasia de bajo grado cada año y para la de alto grado cada 3 a 6 meses (25). Sin embargo, diferentes autores proponen la remoción quirúrgica en los casos de displasia de bajo o de alto grado, aunque el refinamiento y desarrollo de las técnicas de terapia endoscópica han logrado impactar favorablemente el problema (26-28).

La RFA es uno de los métodos más recientemente descrito para el manejo del EB. Datos de dos grandes ensayos clínicos sugieren una tasa de éxito de $92 \%$ a 5 años, incluso en pacientes con solo metaplasia sin displasia $(25,29)$. Para pacientes con bajo grado de displasia se ha mostrado efectiva en $81 \%$ y en $90 \%$ de los casos de displasia de alto grado $(30,31)$. La terapia con RFA es tan efectiva como la resección endoscópica de la mucosa, pero con una menor tasa de complicaciones (13).

Las complicaciones descritas y relacionadas con la RFA son náuseas, dolor torácico, disfagia y odinofagia, ambas transitorias, estenosis, mediastinitis e incluso perforación esofágica que fue manejada con un stent autoexpandible (32-34). La estenosis fue la complicación más consistente en nuestros pacientes y se estima en $8 \%$ de las terapias, lo que es mucho menor que el $88 \%$ descrito después de la resección endoscópica mucosa (35).

No se encontraron complicaciones significativas en el grupo tratado. En buena medida esto es debido al estricto criterio de diferir la terapia en pacientes con esofagitis ulceradas, la realización de la terapia en estos pacientes puede llevar a perforación o a estenosis significativa (36). Sin embargo, recientemente se ha descrito el uso concomitante de la terapia de resección endoscópica de la mucosa en 24 pacientes, seguida por la RFA sin una mayor tasa de complicaciones (37).
Cuando se evalúa el costo-efectividad de la RFA en un estudio con el modelo Markov con una hipotética cohorte de pacientes con 50 años con EB no displásico se consideraron tres escenarios:

1. La historia natural

2. Solo el seguimiento

3. RFA.

Considerando una tasa de erradicación del EB no displásico por RFA de $50 \%$, intencionalmente más baja que lo reportado en los estudios, concluyen que de acuerdo a la edad del paciente, el costo de la RFA y la tasa de erradicación de la RFA, este método es costo efectivo para el EB sin displasia, pero solo dentro de estas condiciones (38).

Un segundo modelo económico fue diseñado para simular la historia natural de una cohorte con EB con edades entre 50 y 80 años o la muerte. Se comparó el costo-efectividad en tres estrategias:

1. Seguimiento

2. Esofagectomía

3. RFA.

La ablación endoscópica por RFA del EB con displasia de alto grado incrementa la expectativa de vida en tres años con un costo menor a $\$ 6000$ dólares comparado con la no intervención. La RFA es la estrategia más costo-efectiva en pacientes con displasia de bajo grado o sin displasia si la ablación erradica permanentemente la displasia de bajo grado en más de $28 \%$ o el EB sin displasia en más de $40 \%$. Para que la RFA sea costo-efectiva, el seguimiento debe ser suspendido después de la terapia (39).

Un tercer modelo utilizó una cohorte de pacientes con 50 años con EB con y sin displasia, seguidos hasta los 80 años o la muerte. Se evaluó el costo-efectividad en tres escenarios:

1. Seguimiento endoscópico con cirugía si se detectaba cáncer 
2. Seguimiento endoscópico con RFA si se detectaba displasia

3. RFA inicial seguida por controles endoscópicos. El estudio encontró que entre los pacientes con displasia de alto grado, la RFA inicial seguida por seguimiento endoscópico fue más costo-efectiva que las otras opciones planteadas (40).

La RFA ha recibido la aprobación para el tratamiento del EB por la FDA en 2005 para el $\mathrm{HALO}^{360}$ y en 2006 para el $\mathrm{HALO}^{90}$. La evidencia científica fue considerada de suficiente calidad para concluir sobre su beneficio en la salud y en la erradicación del epitelio con alto riesgo. Esta tecnología ha sido comparada con el seguimiento endoscópico en el caso de displasia de bajo grado demostrando un mejor pronóstico y una mayor costo-efectividad en el grupo tratado con RFA (13). Además, el beneficio neto de la erradicación completa del EB puede ser logrado por fuera de estudios de investigación formales. Ante esto, la Asociación Americana de Gastroenterología (AGA) recomienda el procedimiento y considera la RFA necesaria en el tratamiento del EB displásico (29), así como en las guías británicas para el manejo del EB (41).

En un reciente estudio retrospectivo con 417 pacientes se demostró que la mayor experiencia con el procedimiento se relacionaba con unos mejores resultados y no es claro cuál es ese número que avala una adecuada experiencia en el procedimiento (42).

Una de las principales preocupaciones con la terapia del EB con RFA es la durabilidad de esta en el tiempo, con una alta heterogeneidad un metanálisis de 18 estudios con 3.802 pacientes que evalúa la eficacia y 6 estudios con 540 pacientes que valora la durabilidad demuestra que la erradicación completa del EB con displasia es de $91 \%$ y la progresión a cáncer fue de solo $0,7 \%$ y la estenosis fue la complicación más frecuente con 5\% (17).

Al comparar un manejo proactivo con RFA con el expectante en EB con displasia leve, Phoa (43) en un estudio multicéntrico, aleatorizado $1: 1$ y controlado con 136 pacientes, se encontró que la ablación disminuye la progresión a DAG de $26,5 \%$ a $1,5 \%$ con una reducción absoluta de $25 \%$, lo que corresponde a un NNT de 4 . Además, la RFA disminuye el riego de progresión a cáncer de 8,8 a 1,5\%, con una reducción absoluta de 7,4\%. En el grupo tratado se logró la ablación total de $92,6 \%$ de la displasia y $88,2 \%$ de la metaplasia intestinal comparado con 27,9 y $0 \%$ en el grupo control. Los efectos adversos fueron de $19,1 \%$ en el grupo tratado; sin embargo, estos fueron leves. El efecto adverso más común fue la estenosis que se resolvió con una mediana de una dilatación endoscópica.

No obstante las limitaciones del presente estudio, principalmente en lo referente al número de pacientes y al corto tiempo de seguimiento, las presentes cifras y lo informado en la literatura mundial hacen en la actualidad a la RFA, en nuestro medio, una terapia factible y asequible y con un potencial de manejo satisfactorio del EB con displasia de cualquier grado así como del carcinoma in situ. Estudios con mayor número de pacientes y un seguimiento mayor en los cuales se sume información genética y molecular del EB nos permitirán definir cuál es ese grupo de pacientes que más se beneficiará de estas terapias; que si bien son bastantes seguras pueden cursan con morbilidad y altos costos.

\section{CONCLUSIONES}

La RFA es una opción para el tratamiento del paciente con EB y epitelio displásico (bajo y alto grado) al disminuir el riesgo de progresión a malignidad. Esta terapia puede ser usada conjuntamente con la remoción endoscópica en los casos de nódulos o lesiones visibles y displasia o carcinoma in situ. Los efectos adversos son escasos y se encontró estenosis del esófago, dolor torácico, o náuseas y vómito; las complicaciones serias son raras. La terapia supresora ácida postintervención es clave para minimizar las molestias locales y permitir la reepitelialización.

\section{Conflictos de intereses}

Los autores no presentan ningún conflicto de interés.

Este estudio se realizó con el apoyo del Proyecto sostenibilidad de la vicerrectoría de investigación de la Universidad de Antioquia.

\section{REFERENCIAS}

1. de Jonge PJ, van Blankenstein M, Grady WM, Kuipers EJ. Barrett's oesophagus: epidemiology, cancer risk and implications for management. Gut 2014; 63: 191-202.

2. Fitzgerald RC, di Pietro M, Ragunath $\mathrm{K}$, et al. British Society of Gastroenterology guidelines on the diagnosis and management of Barrett's oesophagus. Gut 2014; 63: 7-42.

3. Spechler SJ, Sharma P, Souza RF, Inadomi JM, Shaheen NJ, American Gastroenterological A. American Gastroenterological Association technical review on the management of Barrett's esophagus. Gastroenterology 2011; 140: e18-52; quiz e13.

4. Zhang Y. Epidemiology of esophageal cancer. World journal of gastroenterology : WJG 2013; 19: 5598-606.

5. Sie C, Bright T, Schoeman M, et al. Argon plasma coagulation ablation versus endoscopic surveillance of Barrett's esophagus: late outcomes from two randomized trials. Endoscopy 2013; 45: 859-65.

6. Anders M, Bahr C, El-Masry MA, et al. Long-term recurrence of neoplasia and Barrett's epithelium after complete endoscopic resection. Gut 2014. 
7. Barret M, Pratico CA, Beuvon F, et al. Esophageal circumferential en bloc endoscopic submucosal dissection: assessment of a new technique. Surgical laparoscopy, endoscopy \& percutaneous techniques 2013; 23: e182-7.

8. Gosain S, Mercer K, Twaddell WS, Uradomo L, Greenwald BD. Liquid nitrogen spray cryotherapy in Barrett's esophagus with high-grade dysplasia: long-term results. Gastrointestinal endoscopy 2013; 78: 260-5.

9. Gray J, Fullarton GM. Long term efficacy of Photodynamic Therapy (PDT) as an ablative therapy of high grade dysplasia in Barrett's oesophagus. Photodiagnosis and photodynamic therapy 2013; 10: 561-5.

10. Ertan A, Zaheer I, Correa AM, Thosani N, Blackmon SH. Photodynamic therapy vs radiofrequency ablation for Barrett's dysplasia: Efficacy, safety and cost-comparison. World journal of gastroenterology: WJG 2013; 19: 7106-13.

11. Rey-Ferro M, Pinilla RE. Erradicación total de esófago de Barrett con displasia de bajo y alto grado por medio de terapia combinada con resección mucosa focal con Duette y ablación por radiofrecuencia: Reporte de caso y revisión de la literatura. Rev Col Gastroenterol 2013; 28: 124-32.

12. Bulsiewicz WJ, Shaheen NJ. The role of radiofrequency ablation in the management of Barrett's esophagus. Gastrointestinal endoscopy clinics of North America 2011; 21: 95-109.

13. Shaheen NJ, Sharma P, Overholt BF, et al. Radiofrequency ablation in Barrett's esophagus with dysplasia. The New England journal of medicine 2009; 360: 2277-88.

14. Shaheen NJ, Overholt BF, Sampliner RE, et al. Durability of radiofrequency ablation in Barrett's esophagus with dysplasia. Gastroenterology 2011; 141: 460-8.

15. Phoa KN, Pouw RE, van Vilsteren FG, et al. Remission of Barrett's esophagus with early neoplasia 5 years after radiofrequency ablation with endoscopic resection: a Netherlands cohort study. Gastroenterology 2013; 145: 96-104.

16. Orman ES, Kim HP, Bulsiewicz WJ, et al. Intestinal metaplasia recurs infrequently in patients successfully treated for Barrett's esophagus with radiofrequency ablation. The American journal of gastroenterology 2013; 108: 187-95; quiz 96.

17. Orman ES, Li N, Shaheen NJ. Efficacy and durability of radiofrequency ablation for Barrett's Esophagus: systematic review and meta-analysis. Clinical gastroenterology and hepatology : the official clinical practice journal of the American Gastroenterological Association 2013; 11: 124555.

18. Pouw RE, Gondrie JJ, Rygiel AM, et al. Properties of the neosquamous epithelium after radiofrequency ablation of Barrett's esophagus containing neoplasia. The American journal of gastroenterology 2009; 104: 1366-73.

19. Anand O, Wani S, Sharma P. When and how to grade Barrett's columnar metaplasia: the Prague system. Best practice \& research Clinical gastroenterology 2008; 22: 661-9.

20. Smith CD, Bejarano PA, Melvin WS, Patti MG, Muthusamy R, Dunkin BJ. Endoscopic ablation of intestinal metaplasia containing high-grade dysplasia in esophagectomy patients using a balloon-based ablation system. Surgical endoscopy 2007; 21: 560-9.

21. Dunkin BJ, Martinez J, Bejarano PA, et al. Thin-layer ablation of human esophageal epithelium using a bipolar radiofrequency balloon device. Surgical endoscopy 2006; 20: 125-30.

22. Pouw RE, Sharma VK, Bergman JJ, Fleischer DE. Radiofrequency ablation for total Barrett's eradication: a description of the endoscopic technique, its clinical results and future prospects. Endoscopy 2008; 40: 1033-40.

23. Chadwick G, Groene O, Markar SR, Hoare J, Cromwell D, Hanna GB. Systematic review comparing radiofrequency ablation and complete endoscopic resection in treating dysplastic Barrett's esophagus: a critical assessment of histologic outcomes and adverse events. Gastrointestinal endoscopy 2014.

24. Singh M, Gupta N, Gaddam S, et al. Practice patterns among U.S. gastroenterologists regarding endoscopic management of Barrett's esophagus. Gastrointestinal endoscopy 2013;78:689-95.

25. Fleisher LA. Improving perioperative outcomes: my journey into risk, patient preferences, guidelines, and performance measures: Ninth Honorary FAER Research Lecture. Anesthesiology 2010; 112: 794-801.

26. Qumseya BJ, Wang H, Badie N, et al. Advanced imaging technologies increase detection of dysplasia and neoplasia in patients with Barrett's esophagus: a meta-analysis and systematic review. Clinical gastroenterology and hepatology: the official clinical practice journal of the American Gastroenterological Association 2013; 11: 1562-70 e1-2.

27. Gaddam S, Wani S. Endoscopic therapy of Barrett esophagus. Gastrointestinal endoscopy clinics of North America 2013; 23: 1-16.

28. Wu J, Pan YM, Wang TT, Gao DJ, Hu B. Endotherapy versus surgery for early neoplasia in Barrett's esophagus: a metaanalysis. Gastrointestinal endoscopy 2013.

29. Lyday WD, Corbett FS, Kuperman DA, et al. Radiofrequency ablation of Barrett's esophagus: outcomes of 429 patients from a multicenter community practice registry. Endoscopy 2010; 42: 272-8.

30. Ganz RA, Overholt BF, Sharma VK, et al. Circumferential ablation of Barrett's esophagus that contains high-grade dysplasia: a U.S. Multicenter Registry. Gastrointestinal endoscopy 2008; 68: 35-40.

31. Sharma VK, Wang KK, Overholt BF, et al. Balloon-based, circumferential, endoscopic radiofrequency ablation of Barrett's esophagus: 1-year follow-up of 100 patients. Gastrointestinal endoscopy 2007; 65: 185-95.

32. Fleischer DE, Overholt BF, Sharma VK, et al. Endoscopic ablation of Barrett's esophagus: a multicenter study with 2.5-year follow-up. Gastrointestinal endoscopy 2008; 68: 867-76.

33. Yoon SS, Rivera R, Antignano L, Kaul V. A case of mediastinitis after radiofrequency ablation for Barrett's esophagus. Gastrointestinal endoscopy 2011; 74: 1407-8. 
34. Vahabzadeh B, Rastogi A, Bansal A, Sharma P. Use of a plastic endoprosthesis to successfully treat esophageal perforation following radiofrequency ablation of Barrett's esophagus. Endoscopy 2011; 43: 67-9.

35. Chennat J, Konda VJ, Ross AS, et al. Complete Barrett's eradication endoscopic mucosal resection: an effective treatment modality for high-grade dysplasia and intramucosal carcinoma--an American single-center experience. The American journal of gastroenterology 2009; 104: 2684-92.

36. Dabrowski WP, Szczepanik AB, Misiak A, Pielacinski K. Radiofrequency ablation in the management of Barrett's esophagus - preliminary own experience. Wideochirurgia $\mathrm{i}$ inne techniki malo inwazyjne = Videosurgery and other miniinvasive techniques / kwartalnik pod patronatem Sekcji Wideochirurgii TChP oraz Sekcji Chirurgii Bariatrycznej TChP 2013; 8: 107-11.

37. van Vilsteren FG, Alvarez Herrero L, Pouw RE, et al. Radiofrequency ablation and endoscopic resection in a single session for Barrett's esophagus containing early neoplasia: a feasibility study. Endoscopy 2012; 44: 1096-104.

38. Das A, Wells C, Kim HJ, Fleischer DE, Crowell MD, Sharma VK. An economic analysis of endoscopic ablative therapy for management of nondysplastic Barrett's esophagus. Endoscopy 2009; 41: 400-8.

39. Inadomi JM, Somsouk M, Madanick RD, Thomas JP, Shaheen NJ. A cost-utility analysis of ablative therapy for Barrett's esophagus. Gastroenterology 2009; 136: 2101-14 e1-6.

40. Hur C, Choi SE, Rubenstein JH, et al. The cost effectiveness of radiofrequency ablation for Barrett's esophagus. Gastroenterology 2012; 143: 567-75.

41. Fitzgerald RC, di Pietro M, Ragunath K, et al. British Society of Gastroenterology guidelines on the diagnosis and management of Barrett's oesophagus. Gut 2014; 63: 7-42.

42. Fudman DI, Lightdale CJ, Poneros JM, et al. Positive correlation between endoscopist radiofrequency ablation volume and response rates in Barrett's esophagus. Gastrointestinal endoscopy 2014.

43. Phoa KN, van Vilsteren FG, Weusten BL, et al. Radiofrequency ablation vs endoscopic surveillance for patients with Barrett esophagus and low-grade dysplasia: a randomized clinical trial. JAMA: the journal of the American Medical Association 2014; 311: 1209-17. 